\title{
Anatomically Constrained Deformation for Design of Cranial Implant: Methodology and Validation
}

\author{
Ting $\mathrm{Wu}^{1}$, Martin Engelhardt ${ }^{2}$, Lorenz Fieten ${ }^{1}$, \\ Aleksandra Popovic ${ }^{1}$, and Klaus Radermacher ${ }^{1}$ \\ ${ }^{1}$ Helmholtz Institute for Biomedical Engineering, RWTH Aachen University, \\ Aachen, Germany \\ \{twu, fieten, popovic, radermacher\}@hia.rwth-aachen.de \\ ${ }^{2}$ Clinic for Neurosurgery, Ruhr-University, Bochum, Germany \\ martin.engelhardtaruhr-uni-bochum. de
}

\begin{abstract}
We present a new approach for cranial implant design which uses anatomical constrained deformation based on reference models. The methodological framework contains three steps: patient-specific generation of the reference model containing the anatomical constraints about the skull shape; determination of the spatial correspondence between the patient skull and the reference model by 3D matching; adaptive deformation of the fragment on the reference model corresponding to the defect area on the patient skull for implant design. The proposed method was validated by simulating the reconstruction of artificially generated defects on healthy skulls. The validation results show that this approach can generate implant geometry very fast and with satisfactory quality. This approach also outperforms the surface interpolation method in reconstructing cranial defects.
\end{abstract}

\section{Introduction}

Calvarial tumors, infectious bone flap, and neurosurgical external decompression are the main causes for large cranial defects. The main indications for cranial reconstruction of these patients are cosmetic reasons and protection of intracranial structures from mechanical impact [1][2]. With the advance of the three-dimensional (3-D) Computed Tomography (CT) it is possible to reconstruct the 3D patient anatomy and design an implant with help of computers. The currently well-established approaches for implant design often involve a complicated CAD/CAM process chain [3][4][5][6]. In the CAD/CAM based approaches a surgeon needs support from technical staffs or tools out of clinics, which sometimes leads to high cost and low efficiency. Despite the technical improvement in CT and CAD/CAM, the reconstruction of a large-format skull defect sometimes remains difficult because manual construction and modelling of a free-form surface is necessary for each individual patient. Some alternative methods have been proposed to design the implant based on CT data without CAD process by using methods like mirroring [7], surface interpolation or deformation [8][9]. These methods use either 
anatomical or mathematical features of the skull surface to reconstruct the defect area. The mirroring method uses the anatomical features on the contra-lateral part but is suitable for the defect on one side of the skull (unilateral defect) only. The interpolation and deformation methods, such as thin-plate spline (TPS), use mathematical constraints (e.g. $C^{l}$ continuity) to design the implant from the remaining part of a skull, however, without considering anatomical constraints.

In this paper, we present a novel approach for cranial implant design which uses anatomically constrained adaptive deformation based on a reference model. The patient-specific reference model is used as anatomical constraints for implant design. The approach was validated by simulating the reconstruction of 44 artificial defects on nine healthy skulls with known ground truth. Furthermore, we also compare the performance of the proposed method with the surface interpolation method.

\section{Methods}

The methodological framework of anatomically constrained deformation for implant design can be divided into three steps (Fig. 1.): Firstly, a reference model is generated according to the patient skull; then 3D-matching is performed to determine the spatial correspondence between the patient skull and the reference model; finally, adaptive deformation is used to design the implant geometry according to the defect area on the patient skull and its corresponding fragment on the reference model.

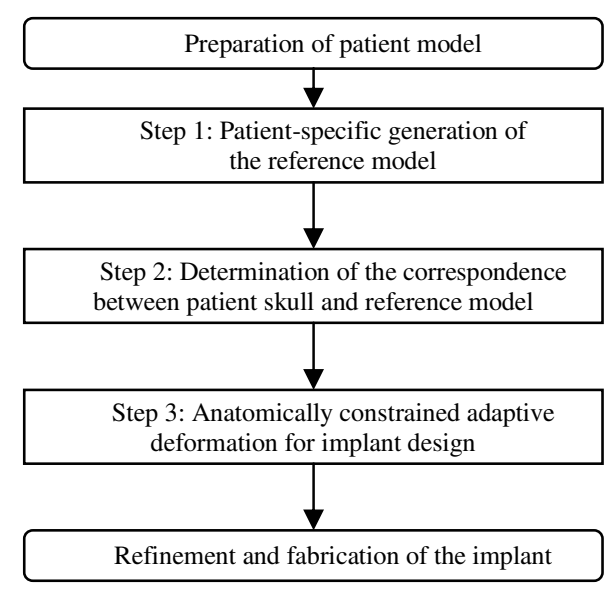

Fig. 1. The methodological framework for anatomically constrained implant design based on reference models in the workflow of cranial implant design and fabrication

\subsection{Patient-Specific Generation of the Reference Model}

The reference model can be generated in different ways according to the patient skull defect. For unilateral defects, mirroring the normal side could be used to 
generate the reference model. For cranial defects spanning both sides of the skull (bilateral defects), or more generally (i.e. also applied to unilateral cases), the reference model can be obtained by searching a similar skull in a 3D reference database of healthy skulls. The reference database contains currently 25 healthy skulls (13 Male, 12 Female) with mean age 36 and median age 39 (range 20-62 years). To enable the efficient 3D retrieval of the similar skull model, the original datasets are indexed by descriptors about the demographic information (e.g. age, sex, etc.) and geometrical characters based on anthropometric measurement of craniofacial landmarks [10]. The craniofacial landmarks are also used to define standardized cutting planes or view directions. The contour of a $2 \mathrm{D}$ images on such planes or in such view directions is then extracted and represented by morphological descriptors based on normalized Fourier descriptors, which enable a similarity comparison with scaling and rotation invariance.

\subsection{Determination of the Spatial Correspondence by 3D Matching}

The paired point matching method [11] was used to match craniofacial landmarks on the patient skull and the corresponding landmarks on the reference model. It is also possible to do the matching based on the user-defined points on the skull surface through user interaction. Through geometric matching, a spatial correspondence between the points of the patient skull and the points of the reference model is established. The aim of this step is to obtain an estimation of transformational relationship between the patient skull and the reference model. Since the shapes of the patient skull and the reference model might be totally different, surface-based matching methods like ICP [12] are not used here. Moreover, the paired point matching method is computationally more efficient for our purpose.

\subsection{Implant Design Based on Anatomically Constrained Adaptive Deformation}

The main idea of the proposed method is to use the reference model as anatomical constraint to control the deformation adaptively at each point. An internal coordinate system based on the craniofacial landmarks is defined so that a point on the surface of the patient skull and the reference model can be described in spherical form $r_{p a t}(\varphi, \theta)$ and $r_{r e f}(\varphi, \theta)$ (i.e. radial distance as function of the directional angles: $\varphi$ and $\theta$ ). The origin of the coordinate system is defined as the centroid of the landmark pair Porion (po, the most superior point on the upper margin of the external auditory meatus when the head is in the Frankfort horizontal plane). The directions of the three axes in Cartesian coordinate system are defined as follows: the average of the directional vectors of the 2 connecting lines between landmarks Porion (po) and Frontozygomaticus (fz, the most lateral points on the frontozygomatic suture) on the left and on the right side, the directional vector along the connecting line from Porion (po) on the right side to Porion (po) on the left side, and their cross product [13].

The implant geometry is generated by adaptive deformation based on radial scaling. The direction-dependent radial scaling factor $s(\varphi, \theta)$ is defined as: 


$$
s(\varphi, \theta)=r_{p a t}(\varphi, \theta) / r_{r e f}\left(\varphi^{\prime}, \theta^{\prime}\right)
$$

where the $\varphi$ and $\theta$ are the directional angle in the coordinate system of the patient skull and the $\varphi^{\prime}$ and $\theta^{\prime}$ are the corresponding directional angle in the coordinate system of the reference model calculated by using the transformational relation determined in the step 2. By user-interactive delineation of the defect margin, a set of points on the patient skull $\left\{r_{p a t}\left(\varphi_{i}, \theta_{i}\right), i=1, \ldots, N\right\}$ are defined. Since the radial distance of their corresponding points on the reference model can be determined, the scaling factor $s\left(\varphi_{i}, \theta_{i}\right)$ at each point $r_{p a t}\left(\varphi_{i}, \theta_{i}\right)$ is known. Taking all these points as control points, the scaling factor $s(\varphi, \theta)$ on the defect area of the patient skull (i.e. where $r_{p a t}(\varphi, \theta)$ is unknown) can be estimated by interpolation according to directional angles. Since the radial distance on the fragment of the reference model corresponding to the defect area of the patient skull is known, the radial distance at the defect area can be estimated by scaling the radial distance on the reference model with the interpolated scaling factor. In this way, the coordinates of the surface points of the implant to reconstruct the defect can be calculated. So the implant surface is the result of deforming the corresponding fragment on the reference model by radial adaptive scaling. The thickness of the implant can be set by users. In contrast to the direct surface interpolation method, we first interpolate the scaling factor and then multiply the scaling factor with the radial distance on the reference model so that both the remaining part of the patient skull and the reference model (which contains anatomical constraints) contribute to the final implant geometry (Fig. 2.).

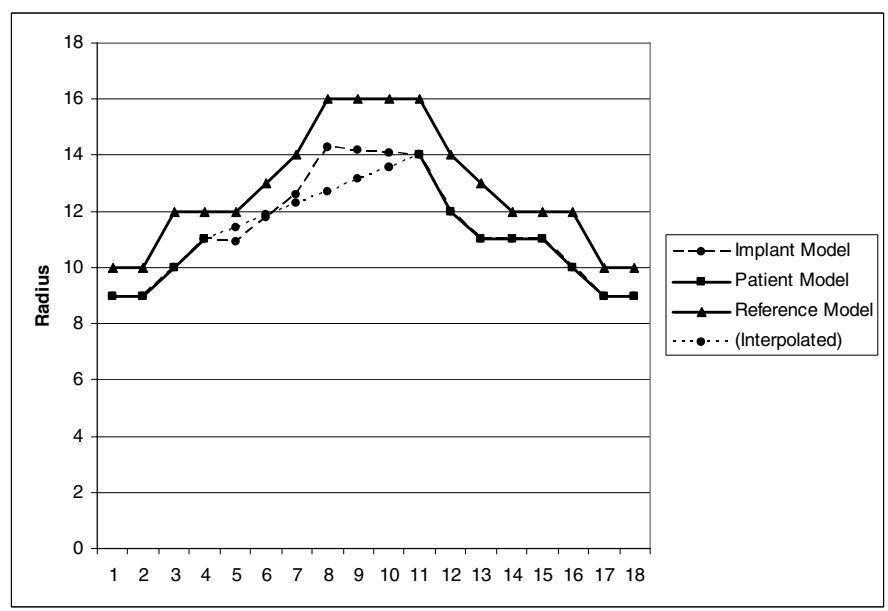

Fig. 2. Illustration of the anatomically constrained deformation. The reference model gives the anatomical constrain for implant geometry while direct interpolation fails to recover certain anatomical detail on the defect area. 
Further refinement such as morphological operation is used to smooth the implant and make it fit well with the defect on the patient skull.

\section{Results}

To validate the proposed methods, nine datasets of healthy skull were selected and totally 44 defects with different size and location were generated from these datasets. Since the ground truth is known, we can evaluate the quality of the reconstruction. We also compared the proposed method with the surface interpolation method.

\subsection{Computational Performance}

The time needed for the entire reconstruction process is less than 25 minutes on a Pentium 4 computer $(3.2 \mathrm{GHz}, 1 \mathrm{~GB}$ RAM). Compared with the CAD/CAM based approaches which might need a couple of days, it is now possible for the surgeon to perform the planning task of implant design on his own.

\subsection{Validation of the Reconstruction Quality with Ground Truth}

The reconstruction quality was evaluated by comparing the healthy skull (ground truth) and the test datasets (i.e. the original healthy skull with an artificial defect) reconstructed with the implant designed with the proposed method. Taking 9 healthy skulls (5 Male, 4 Female, Age range 26-47) as ground truth, 44 test datasets are generated by artificially removing a fragment from one of the healthy skulls. These 9 healthy skulls were not contained in the reference database. The defects have different sizes and different locations. The assessment criterion of the reconstruction quality is the reconstruction error defined as the difference between radial distance of surface voxels on the original skull (ground truth) and radial distance of surface voxels on the reconstructed part of the test datasets (i.e. implant). The reconstruction error is measured in voxels so that this criterion is independent of the resolution of CT. The average percentage of surface voxels with reconstruction error within certain ranges (e.g. error $<1$ voxel) were calculated for statistical evaluation.

Table 1. The reconstruction quality of the implant measured by the difference in the radial distance: the distribution of the reconstruction error within different range

\begin{tabular}{lllr}
\hline & $0-1$ voxel & $2-4$ voxels & $>4$ voxels \\
\hline Total (44) & $81.6 \%(68 \%-96 \%)$ & $12.8 \%(4 \%-19 \%)$ & $5.6 \%(0 \%-13 \%)$ \\
Group A (21) & $82.8 \%$ & $12.0 \%$ & $5.2 \%$ \\
Group B (23) & $80.5 \%$ & $13.5 \%$ & $6.0 \%$ \\
Group 1 (16) & $88.0 \%$ & $9.0 \%$ & $3.0 \%$ \\
Group 2 (28) & $77.9 \%$ & $15.1 \%$ & $7.1 \%$ \\
\hline
\end{tabular}

An average of $81.6 \%(68 \%-96 \%)$ of the surface points can be reconstructed within an error of 1 voxel. The average percentage of surface points reconstructed 
with large error (>4 voxels) is less than 6\% (Table 1.). According to the defect size, the test datasets are subdivided into 2 groups: Group A covering less than $100 \mathrm{~cm}^{2}$ $(\mathrm{n}=21)$, Group B covering more than $100 \mathrm{~cm}^{2}(\mathrm{n}=23)$. There is no significant difference between these two groups considering the reconstruction quality. The test datasets can also be subdivided into 2 groups according to coverage and location: Group 1 with unilateral defect $(n=16)$ and Group 2 with bilateral defect $(n=28)$. There is a significant difference between these two groups because the mirrored model provides more patient-specific and precise anatomical constraints for implant design. This can be further confirmed when implants are designed using reference skull models instead of mirrored models for unilateral defects (Table 2.).

Table 2. Comparison of reconstruction quality of the implant for unilateral defects (Total 16) using mirrored models (Method 1) and similar skulls as reference models (Method 2)

\begin{tabular}{llcr}
\hline & $0-1$ voxel & $2-4$ voxels & $>4$ voxels \\
\hline Method 1 & $88.0 \%$ & $9.0 \%$ & $3.0 \%$ \\
Method 2 & $79.5 \%$ & $14.8 \%$ & $5.7 \%$ \\
\hline
\end{tabular}

It is also shown that the reconstruction quality is strongly dependent on the availability of similar skulls in the reference database when reconstructing bilateral defects (Table 3.). However, if the reference database contains skulls with large varieties, good results could be expected in general.

Table 3. Comparison of reconstruction quality of the implant for skull defects (Total 20) using the most similar skull (Method 1) and using a skull by randomized selection in the reference skull database (Method 2) as the reference model. Method 2 has a large deviation in reconstruction error compared with Method 1.

\begin{tabular}{lllr}
\hline & $0-1$ voxel & $2-4$ voxels & $>4$ voxels \\
\hline Method 1 & $79.6 \%(68 \%-93 \%)$ & $14.1 \%(6 \%-19 \%)$ & $6.3 \%(1 \%-13 \%)$ \\
Method 2 & $62.3 \%(38 \%-87 \%)$ & $27.2 \%(10 \%-46 \%)$ & $10.5 \%(3 \%-29 \%)$ \\
\hline
\end{tabular}

\subsection{Comparison to Surface Interpolation Methods}

The proposed method has been compared with the surface interpolation method using thin-plate splines [8]. Twelve test datasets were reconstructed by these two methods. It is shown that our method outperforms the surface interpolation method. Although the surface interpolation method can recover a smooth surface on the defect area, it has no anatomical constraints which can contribute to a better reconstruction quality for individual patients (Fig.3. and Table 4.). For the surface interpolation method, the surface voxels near to the defect margin have usually less reconstruction error whereas the surface voxels at the middle part of the defect area have often the large reconstruction error. This shows the limitation of pure mathematical constraints for implant design. 
Table 4. Comparison of reconstruction quality of 12 test datasets between the proposed method (Method 1) and the surface interpolation method (Method 2)

\begin{tabular}{lllr}
\hline & $0-1$ voxel & $2-4$ voxels & $>4$ voxels \\
\hline Method 1 & $81.0 \%$ & $13.1 \%$ & $5.9 \%$ \\
Method 2 & $69.5 \%$ & $18.8 \%$ & $11.7 \%$ \\
\hline
\end{tabular}

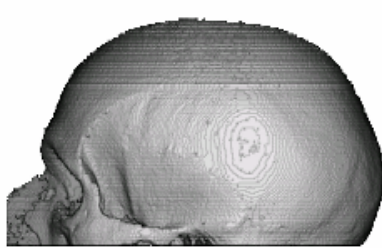

(A)

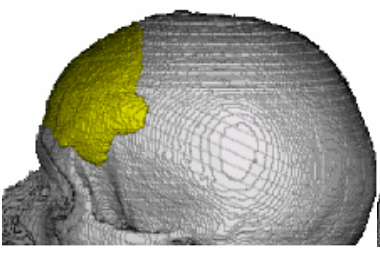

(B)

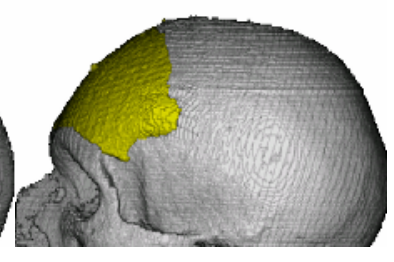

(C)

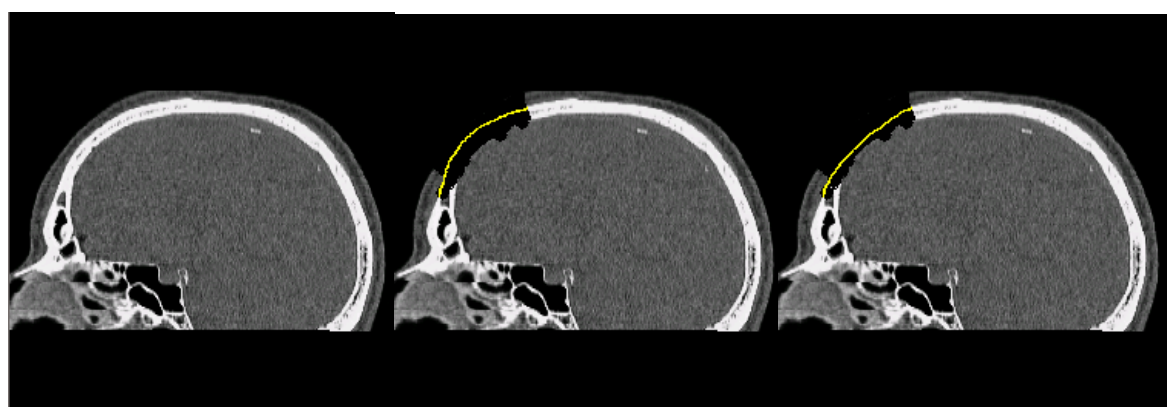

(D)

(E)

(F)

Fig. 3. Comparison of the reconstruction using anatomically constrained deformation and surface interpolation with thin-plate splines. (A) Original dataset (ground truth) (B) Reconstruction with anatomically constrained deformation (the yellow part is implant) (C) Reconstruction with thin-plate splines (D) Midsagittal slice of the original dataset (E) Midsagittal slice of reconstruction with anatomically constrained deformation (F) Midsagittal slice of reconstruction with thin-plate splines.

\section{Conclusions}

In this paper, a new approach to design cranial implant using anatomically constrained deformation is presented and validated by reconstruction simulated skull defects with known ground truth. The results show that the proposed method is fast and can design implants with quite good quality. Therefore, the surgeon can perform the task of designing the implant geometry without the help of technicians and use the original CT data for medical verification in one planning session. 
Besides the clinical studies, the ongoing work includes introducing statistical shape models of skull in this methodological framework and investigation of the influence of the user-interactive localization of craniofacial landmarks and the defect margin to the reproducibility of the implant design. Furthermore, the database structure and its search strategies will be extended and optimized for an internet-based architecture so that the central reference database of the skull geometry can be shared by different users.

\section{References}

1. Sanan A, Haines SJ.: Repairing holes in the head: a history of cranioplasty. Neurosurgery 40 (1997) 588-603

2. Dujovny M, Aviles A, Agner C, Fernandez P, Charbel FT.: Cranioplasty: cosmetic or therapeutic? Surg Neurol 47 (1997) 238-241

3. Eufinger H, Wehmöller M, Machtens E, Heuser L, Harders A, Kruse D. Reconstruction of craniofacial bone defects with individual alloplastic implants based on CAD/CAMmanipulated CT-data. J Cranio Maxillofac Surg 23 (1995) 175-181

4. Heissler E, Fischer FS, Bolouri S, Lehmann T, Mathar W, Gebhardt A, et al. Custom-made cast titanium implants produced with $\mathrm{CAD} / \mathrm{CAM}$ for the reconstruction of cranium defects. Int J Oral Maxillofac Surg 27 (1998) 334-338

5. Lee MY, Chang CC, Lin CC, Lo LJ, Chen YR. Custom implant design for patients with cranial defects. IEEE Eng Med Biol Mag 21(2) (2002) 38-44

6. Müller A, Krishnan KG, Uhl E, Mast G. The application of rapid prototyping techniques in cranial reconstruction and preoperative planning in neurosurgery. J Craniofac Surg $\mathbf{1 4}$ (2003) 899-914

7. Sauret V, Linney AD, Richards R. Computer assisted surgery: the use of digital images in enabling computerized design and manufacture of titanium implants. Imaging 14 (2002) 464-471

8. Carr JC, Fright WR, Beatson RK. Surface interpolation with radial basis functions for medical imaging. IEEE Transactions in Medical Imaging 16(1) (1997) 96-107

9. Min KJ., Dean D.: Highly accurate CAD tools for cranial implants. In Ellis, R.E. and Peters, T.M., eds.: Proceedings of Sixth International Congerence on Medical Image Computing and Computer Assisted Intervention, MICCAI 2003. Volume 2878 of Lecture Notes on Computer Science., Springer-Verlag (2003) 99-107

10. Kolar JC, Salter EM. Craniofacial anthropometry practical measurements of the head and face for clinical, surgical and research use. Charles Thomas publisher ltd., USA (1996).

11. Horn BKP. Closed-form solution of absolute orientation using unit quaternions. J. Opt. Soc. Am. A 4(4) (1987) 629-642

12. Besl PJ, McKay ND. A method for registration of 3-d shapes. IEEE Transactions on Pattern Analysis and Machine Intelligence 14(2) (1992) 239-256 